\title{
Evaluation of the anastomoses between the ophthalmic artery and the middle meningeal artery by superselective angiography
}

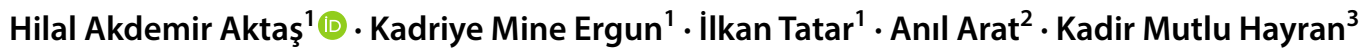

Received: 4 June 2020 / Accepted: 6 August 2020 / Published online: 16 August 2020

(c) Springer-Verlag France SAS, part of Springer Nature 2020

\begin{abstract}
Purpose There are three anastomoses between the ophthalmic artery (OA) and the middle meningeal artery (MMA): the anastomotic branch with MMA, the recurrent meningeal branch and the anterior falx artery. We aimed to evaluate the anastomotic branches between the OA and the MMA on superselective angiograms of pediatric patients with retinoblastoma.

Materials and methods We evaluated 126 angiographies performed on children with retinoblastoma. The mean diameter and angiographic visibility percentage of the anastomotic branches between the OA and the MMA were examined according to age group and sex.

Results The mean diameter of anastomotic branch with MMA was measured $0.58 \pm 0.13 \mathrm{~mm}$ and we found this branch in 15 of 126 angiographic images (11.9\%). We detected the recurrent meningeal branch in 47 of total images (37.3\%). The recurrent meningeal branch arose $85.1 \%$ from the lacrimal artery, $8.5 \%$ from the anastomotic branch with MMA and 6.4\% directly from the OA. The mean diameter of this artery was measured $0.21 \pm 0.06 \mathrm{~mm}$. Anterior falx artery was found in 86 of 126 angiographic peocedures $(68.3 \%)$ and the mean diameter was measured $0.22 \pm 0.06 \mathrm{~mm}$.

Conclusion Knowledge of the anastomoses between the OA and the MMA system are all necessary to perform safe and successful endovascular and surgical procedures involving the orbital region.
\end{abstract}

Keywords Ophthalmic artery $\cdot$ Middle meningeal artery $\cdot$ Anastomoses $\cdot$ Superselective angiography

\section{Introduction}

The main blood supplying to the orbital structures is the ophthalmic artery (OA) and its branches. The OA is a branch of the internal carotid artery (ICA) and it enters the orbit through the optic canal. The main branches of the OA are the central retinal artery, ciliary arteries, lacrimal artery (LA), muscular branches, supraorbital artery, anterior and posterior ethmoidal arteries, meningeal branch, medial palpebral arteries, supratrochlear artery and dorsal nasal artery [1]. The most crucial collateral blood supply to the orbit is the middle meningeal artery (MMA) [2].

Hilal Akdemir Aktaş

hakdemir06@hotmail.com

1 Department of Anatomy, Faculty of Medicine, Hacettepe University, 06100 Ankara, Turkey

2 Department of Radiology, Faculty of Medicine, Hacettepe University, 06100 Ankara, Turkey

3 Department of Preventive Oncology, Faculty of Medicine, Hacettepe University, 06100 Ankara, Turkey
The MMA is a branch of the external carotid artery and it enters the cranium through the foramen spinosum and runs in the groove of the squamous part of the temporal bone [1, 3]. On the greater wing of the sphenoid bone, the MMA divides into anterior and posterior branches [4]. Particularly, anterior branch of the MMA contributes to the blood supply of the orbital region through the superior orbital fissure and/ or Hyrtl canal [2]. There are three anastomoses between the OA and the MMA as follows: the anastomotic branch with MMA, the recurrent meningeal branch, and the anterior falx artery (Fig. 1) [5, 6].

The anastomotic branch with MMA is a branch of the LA or the OA and anastomose with the anterior division of the MMA through the Hyrtl canal (cranio-orbital foramen) $[2,7]$. The recurrent meningeal branch is a branch of the LA and anastomose with the anterior branch of the MMA through the superior orbital fissure [1]. This branch is seen thiny, tortuous and rarely visible angiographically [8]. It usually contributes a little to the blood supply of the orbit, but in some cases this anastomosis is very large and become main supply of the orbital region [9]. The anterior falx artery is a 


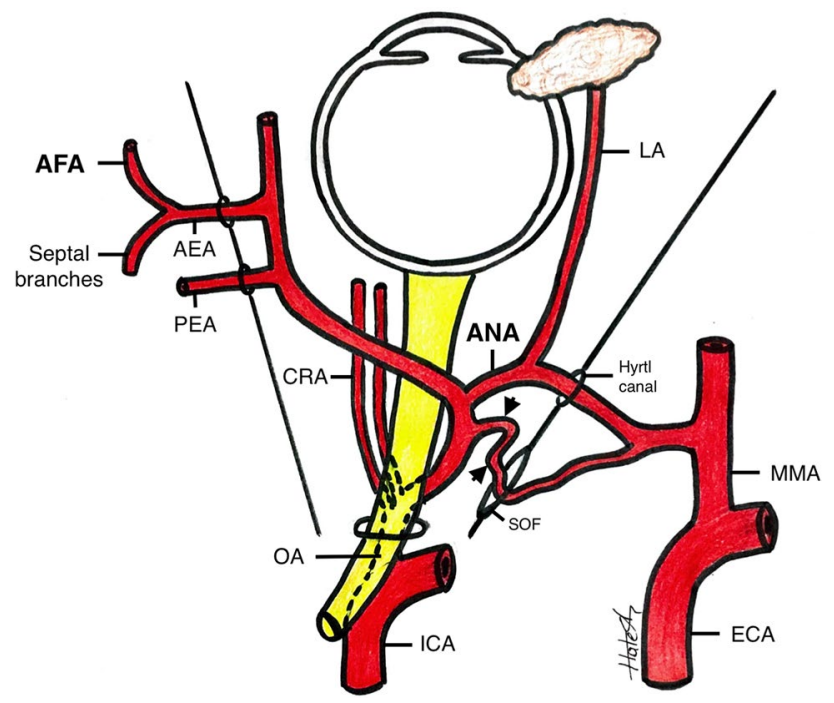

Fig. 1 Schematic drawing shows the three anastomoses between the OA and the MMA. These anastomoses are as follows: the anastomotic branch with MMA (ANA), the recurrent meningeal branch (black arrowheads), and the anterior falx artery (AFA). (AEA anterior ethmoidal artery, CRA central retinal artery, ECA external carotid artery, ICA internal carotid artery, LA lacrimal artery, MMA middle meningeal artery, OA ophthalmic artery, PEA posterior ethmoidal artery, SOF superior orbital fissure)

branch of the anterior ethmoidal artery and anastomose with the anterior branch of the MMA within the falx cerebri [6, 10]. Anterior ethmoidal artery enters the cranium through the anterior ethmoidal foramen and then gives off the anterior falx artery to the dura mater [1,7]. The anastomoses between the OA and the MMA are quite important clinically [11-13]. An endovascular embolization of the MMA carries a risk of blindness due to anastomoses between the MMA and the OA system. Blindness is usually caused by embolic occlusion in the central retinal artery through the anastomotic pathways [14]. Knowledge of the anastomoses between the OA and the MMA system are all necessary to perform safe and successful endovascular and surgical procedures involving the orbit $[11,14]$. The anastomoses between the OA and the MMA depend on the embryological development of the OA [8].

\section{Embryology of the ophthalmic artery}

The OA has a complex embryological development and occurs from three different embryological systems: the primitive ICA, the stapedial artery and the pharyngeal artery systems [5]. The knowledge about the embryological development of the OA based on the studies of Padget [15] and Lasjaunias [7]. At first stages of embryological development, the primitive dorsal and ventral ophthalmic arteries develop from the primitive ICA. In 18-mm embryos, the stapedial artery proceeds with progression and gives off the maxillomandibular and supraorbital branches. The supraorbital branch of the stapedial artery enters the orbit via superior orbital fissure and anastomoses with the primitive OA. This anastomosis between the supraorbital branch and the primitive $\mathrm{OA}$ forms the recurrent meningeal branch in adults. The maxillomandibular branch of the stapedial artery anastomoses with the future external carotid artery and begins to form the MMA. We aimed to redefine the anastomotic branches between the OA and the MMA on superselective angiograms of pediatric patients with retinoblastoma and to review the literature about these anastomotic routes.

\section{Materials and methods}

Superselective OA angiography images, which were obtained from the endovascular procedures of children undergoing intra-arterial chemotherapy for retinoblastoma, were retrospectively analyzed and morphometric measurements were performed. In this cross-sectional retrospective study, superselective angiography images of 126 pediatric patients (age range 4-120 months), who were admitted to the Interventional Radiology Department of Hacettepe University between 2013 and 2017, were evaluated. The study group was divided into six groups according to age as follows: 0-12 months, 13-24 months, 25-36 months, 37-48 months, 49-60 months, and $\geq 61$ months. Because of the effect of chemotherapy on vessel morphometry, the angiographic images obtained from the first endovascular chemotherapy procedure of each patient were included in this study.

Ethical approval for the study was obtained from the ethics committee of Hacettepe University (January 3, 2017, GO 17/22-17). For this type of study formal consent is not required.

\section{General description of the superselective angiography method}

A superselective angiography method was preferred in this study because it is successful in demonstrating vessels smaller than $0.5 \mathrm{~mm}$. Angiography was performed by two endovascular intervention specialists, and patients were treated under general anesthesia and in a $70-100 \mathrm{u} / \mathrm{kg}$ iv heparinized condition. Angiographic images were obtained using a non-ionic water-soluble contrast medium containing 300-350 mg of iodine per milligram with a 4F diagnostic catheter. Two plane (Artis Zeego VC21A / B, Siemens, Erlangen, Germany) angiography units were used. For selective ICA angiogram, a 4-6 ml non-ionic iodinated contrast agent was injected in approximately $2 \mathrm{~s}$, and an image was obtained in the 
anteroposterior and lateral projections. An internal lumen of a 0.0165 inch microcatheter was inserted into the OA with the help of micro guide wire $(0.010$ to 0.014 inch thick guide wire) through the $4 \mathrm{~F}$ catheter. Superselective angiography images were obtained after microcatheter insertion, and $30 \mathrm{ml}$ of melphalan and/or topotecan solution was injected for $30 \mathrm{~min}$. After chemotherapy infusion, the OA and ICA injections were repeated and the anticoagulation was reversed with protamine sulfate.

\section{Image evaluation and analysis method}

Images were obtained from the Hacettepe PACS system and measurements were made with the Osirix program. For all projections, measurements were calibrated with respect to the $4 \mathrm{~F}$ catheter. In order to avoid measuring vessel diameter below the actual value, measurements were done under optimal vascular opacification.

The diameters of the anastomotic branch with MMA and the recurrent meningeal branch were measured on lateral projection images of superselective OA angiography. The diameter of the anterior falx artery was measured on anteroposterior projection of OA superselective angiography.

The parameters evaluated in this study were as follows:

- The origins and diameters (nearest point to the origin) of anastomotic branches between the OA and the MMA.

- Angiographic visibility percentages of anastomotic branches between the OA and the MMA.

\section{Statistical analysis}

IBM SPSS Statistics 23.0 (IBM Corporation, Armonk, NY, USA) was used for statistical analysis. The relationship between variables and age groups was evaluated using the Spearman correlation test, and the relationship between variables and sex was evaluated using the independent groups' student $t$-test. A $p$ value $<0.05$ was considered statistically significant in all statistical evaluations.

\section{Results}

A total of 126 patients ( 54 girls, 72 boys) were included in the study. The age range of subjects were 4-120 months old and the mean age was 32.4 months.

\section{Anastomotic branch with MMA}

In our study, we detected the anastomotic branch with MMA in 15 of 126 angiographic procedures (Fig. 2,3). The angiographic visibility percentages of this artery was calculated $11.9 \%(15 / 126)$. We found that all anastomotic

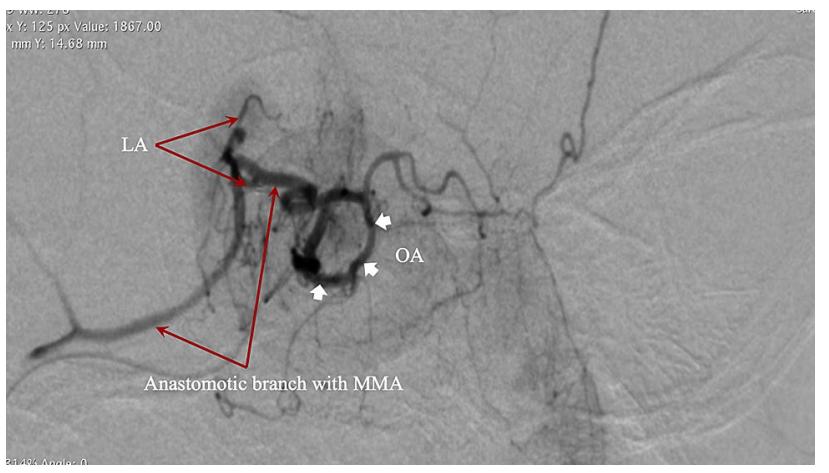

Fig. 2 Superselective angiography of OA in anteroposterior projection shows the anastomotic branch with MMA and the LA. Anastomotic branch with MMA arises from the main trunk of the OA and gives off the LA. (White arrows: OA course, OA ophthalmic artery, LA lacrimal artery, MMA middle meningeal artery)

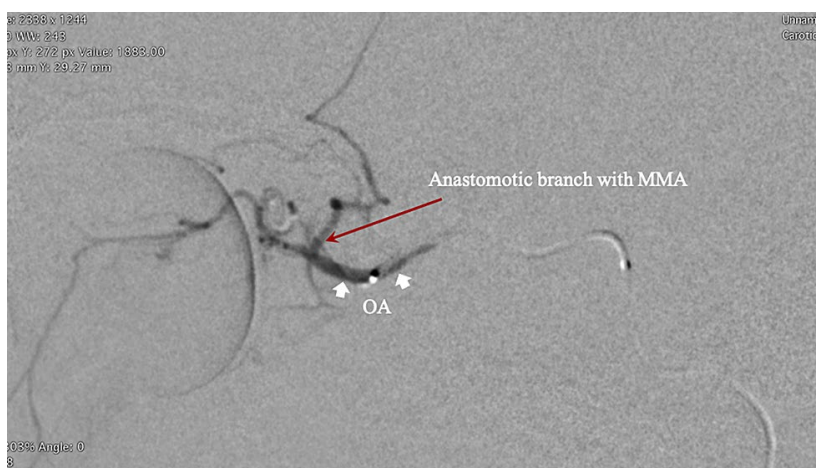

Fig. 3 Superselective angiography of OA in lateral projection shows the OA course and the anastomotic branch with MMA. Anastomotic branch with MMA originates from the main trunk of the OA and courses superiorly to make an anastomosis with the MMA. (White arrows: OA course, OA ophthalmic artery, MMA middle meningeal artery)

branches with MMA arose from the main trunk of the OA. The mean diameter was measured $0.58 \pm 0.13 \mathrm{~mm}$ (min-max $0.32-0.74 \mathrm{~mm}), 0.55 \pm 0.11 \mathrm{~mm}(\min -\max 0.37-0.66 \mathrm{~mm})$ in girls and $0.61 \pm 0.15 \mathrm{~mm}(\min -\max 0.32-0.74 \mathrm{~mm})$ in boys. No statistically significant relationship was found between the mean diameter and sex $(p>0.05)$. We found that 100 of 114 lacrimal arteries $(87.7 \%)$ arose from the main trunk of the OA and 14 (12.3\%) from the anastomotic branch with MMA. Namely, 15 anastomotic branches with MMA gave off 14 lacrimal arteries.

\section{Recurrent meningeal branch}

We detected the recurrent meningeal branch in 47 of 126 angiographic images. The angiographic visibility percentage was found to be $37.3 \%$ (47/126). The recurrent meningeal branch arose $85.1 \%$ from the LA, $8.5 \%$ from the 


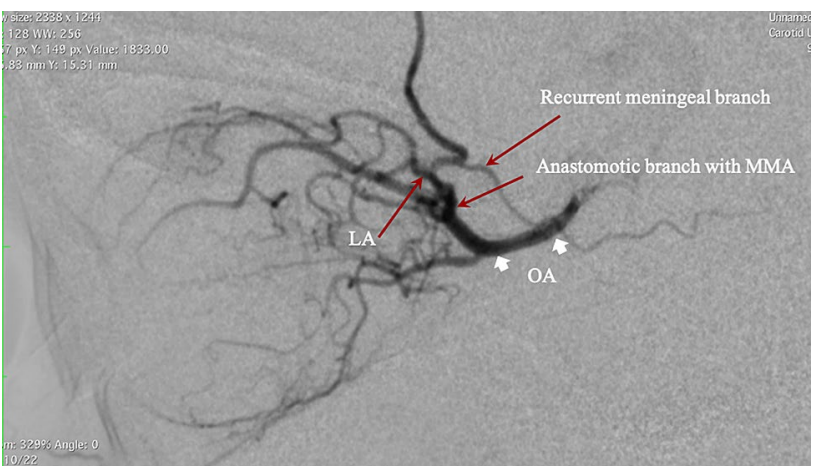

Fig. 4 Superselective angiography of OA in lateral projection shows the OA course, the anastomotic branch with MMA and the recurrent meningeal branch. LA and recurrent meningeal branch originate from the anastomotic branch with MMA. (White arrows: OA course, OA ophthalmic artery, LA lacrimal artery, MMA middle meningeal artery)

Table 1 Morphometry of the recurrent meningeal branch according to age group

\begin{tabular}{lll}
\hline $\begin{array}{l}\text { Age group } \\
\text { (months) }\end{array}$ & \multicolumn{2}{l}{ Recurrent meningeal branch } \\
\cline { 2 - 3 } & Mean \pm SD $(\mathrm{mm})$ & $\begin{array}{l}\text { Angiographic } \\
\text { visibility }(\%)\end{array}$ \\
\hline$\leq 12(n: 35)$ & $0.20 \pm 0.07$ & 50.0 \\
$13-24(n: 31)$ & $0.22 \pm 0.04$ & 28.6 \\
$25-36(n: 29)$ & $0.22 \pm 0.07$ & 46.4 \\
$37-48(n: 13)$ & $0.19 \pm 0.05$ & 23.1 \\
$49-60(n: 8)$ & $0.24 \pm 0.06$ & 37.5 \\
$\geq 61(n: 18)$ & $0.24 \pm 0.03$ & 23.5 \\
General $(n: 126)$ & $0.21 \pm 0.06$ & 37.3 \\
\hline
\end{tabular}

anastomotic branch with MMA and 6.4\% directly from the OA (Fig. 4). The mean diameter of this artery was measured $0.21 \pm 0.06 \mathrm{~mm}$ (min-max: $0.11-0.43 \mathrm{~mm}), 0.22 \pm 0.05 \mathrm{~mm}$ (min-max: $0.15-0.36 \mathrm{~mm}$ ) in girls and $0.21 \pm 0.07 \mathrm{~mm}$ (min-max: 0.11-0.43 mm) in boys. We detected both the recurrent meningeal branch and the anastomotic branch with MMA in only four angiographic procedures. The diameters and the angiographic visibility percentage of the recurrent meningeal branch evaluated by age group are summarized in Table 1. The mean diameter of this branch showed a positive and statistically significant relationship with age $(r=0.29$, $p=0.047)$. As the age increased, the diameters of this artery were found to be increased. There was no statistically significant relationship between the diameter of this branch and $\operatorname{sex}(p>0.05)$.

\section{Anterior falx artery}

We detected the anterior falx artery in 86 of 126 angiographic procedures. The angiographic visibility

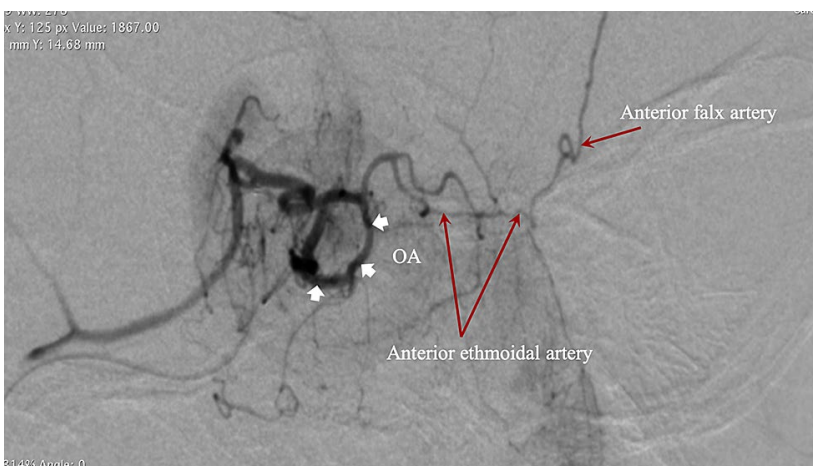

Fig. 5 Superselective angiography of OA in anteroposterior projection shows the anterior ethmoidal artery and the anterior falx artery. After entering the cranium, anterior ethmoidal artery gives off the anterior falx artery inside the falx cerebri. (White arrows: OA course, OA ophthalmic artery)

Table 2 Morphometry of the anterior falx artery according to age group

\begin{tabular}{lll}
\hline $\begin{array}{l}\text { Age group } \\
\text { (months) }\end{array}$ & \multicolumn{2}{l}{ Anterior falx artery } \\
\cline { 2 - 3 } & Mean \pm SD $(\mathrm{mm})$ & $\begin{array}{l}\text { Angiographic } \\
\text { visibility }(\%)\end{array}$ \\
\hline$\leq 12(n: 35)$ & $0.21 \pm 0.04$ & 71.9 \\
$13-24(n: 31)$ & $0.22 \pm 0.06$ & 78.6 \\
$25-36(n: 29)$ & $0.22 \pm 0.07$ & 64.3 \\
$37-48(n: 13)$ & $0.23 \pm 0.05$ & 61.5 \\
$49-60(n: 8)$ & $0.23 \pm 0.06$ & 87.5 \\
$\geq 61(n: 18)$ & $0.25 \pm 0.07$ & 47.1 \\
General $(n: 126)$ & $0.22 \pm 0.06$ & 68.3 \\
\hline
\end{tabular}

percentage of this artery was calculated $68.3 \%(86 / 126)$. We found that all anterior falx arteries arose from the anterior ethmoidal artery (Fig. 5). The mean diameter of this artery was found to be $0.22 \pm 0.06 \mathrm{~mm}$ (min-max $0.12-0.39 \mathrm{~mm}), 0.23 \pm 0.06 \mathrm{~mm}(\min -\max 0.12-0.37)$ in girls and $0.22 \pm 0.06 \mathrm{~mm}(\min -\max 0.12-0.39 \mathrm{~mm})$ in boys. The diameters and the angiographic visibility percentage of this artery evaluated by age group are summarized in Table 2 . There was no statistically significant relationship between the diameter of this branch and sexage $(p>0.05)$.

\section{Discussion}

The OA is a branch of the ICA and it enters the orbit through the optic canal. OA rarely arises from the cavernous segment of the ICA and enters the orbit via the superior orbital fissure. OA also arises from the MMA more frequently without connection to ICA [16]. 
A detailed knowledge of the anastomotic pathways between the OA and the MMA has a major importance for neurosurgeons and neurointerventional radiologists who perform an intervention to the orbital region. The anastomotic branches between the MMA and the OA have been described in the literature but the nomenclature of these branches is highly complicated $[2,17,18]$. We designed this study to clarify this complexity in the literature and to contribute to the literature on pediatric anastomotic routes.

\section{Anastomotic branch with MMA and recurrent meningeal branch}

The anterior division leaves from the MMA within the middle cranial fossa and sends two branches into orbit. One branch enters the orbit through the superior orbital fissure and anastomoses with the "recurrent meningeal branch" [1]. The other branch passes through the Hyrtl canal (cranioorbital foramen) and anastomoses with the "anastomotic branch with MMA" $[2,19]$ or "meningolacrimal artery" $[7$, $8,14,20]$. As a result of dissection of 170 orbits, Hayreh and Dass [21] conveyed the impression that these two transorbital pathways are homologous and reciprocally. However, simultaneous existence of the two arteries indicates that they are not homologous and the two transorbital pathways do not represent alternative routes [2].

According to Lasjaunias, there are two variants of LA collaterals. In the lacrimal variant, the blood supply to the lacrimal gland comes from the OA; the MMA anastomoses with the intraorbital LA through the superior orbital fissure via the recurrent meningeal branch. In the meningolacrimal variant, the lacrimal branch originates directly from the MMA and enters the orbit through the Hyrtl's canal. The MMA anastomoses directly with the OA via the meningoophthalmic artery through the superior orbital fissure [7]. When the meningolacrimal artery supplies entirely the lacrimal region, this artery is quite well visualized angiographically [8]. Perrini et al. [14] dissected 14 adult cadaveric orbits and detected the meningolacrimal artery in only 4 orbits (29\%). They found that the vascularization of the lacrimal gland is provided only by the MMA via the meningolacrimal artery, without contributions from the OA system [14]. Similar to the study of Perrini et al. [14], we detected the anastomotic branch with MMA (meningolacrimal artery) in 15 of 126 angiographic procedures (11.9\%). We determined that the lacrimal region was supplied only by the LA arising from the anastomotic branch with MMA.

Bracco et al. [22] found that this anastomotic branch showed a variable diameter as it could be a very thin artery or such a large artery to represent. The territory supplied by the anastomotic branch and the diameter of this artery depend on the development of the lacrimal branch of the
OA $[8,21]$. In our study, the mean diameter of the anastomotic branch with MMA was measured $0.58 \pm 0.13 \mathrm{~mm}$ and we detected no lacrimal artery originating from the $\mathrm{OA}$ in these cases.

Anastomoses between the recurrent meningeal branch and the MMA are commonly found in anatomical dissections [21]. This anastomosis is formed by the embryological connection between the supraorbital branch of the stapedial artery and the primitive OA [7]. Hayreh and Dass [21] found this anastomosis in all 59 specimens they studied. Similarly, Liu and Rhoton [23] described this anastomotic connection in nine of 10 specimens. Perrini et al. [14] dissected 14 adult cadaveric orbits and detected the recurrent meningeal branch in 10 orbits (71\%). Bracco et al. [22], investigated transorbital anastomotic pathways between external and internal carotid systems in 97 children (106 orbits) affected by intraocular retinoblastoma. They found that the MMA had an anastomosis with the LA in 22 cases $(20.7 \%)$ via recurrent meningeal branch, with the OA in 15 cases (14\%) via meningo-ophthalmic artery and with the supraorbital artery in only one case (0.9\%). Erdoğmuş and Gövsa [24] dissected 38 orbits and detected a recurrent meningeal branch in six orbits $(31.57 \%)$ on the right and five orbits $(26.31 \%)$ on the left. In our study, recurrent meningeal branches were detected in 47 of 126 angiographic images (37.3\%), similar to Bracco et al. [22] and Erdoğmuş and Gövsa's studies [24].

Erdoğmuş and Gövsa [24] determined that all of the recurrent meningeal branches arose from the LA. Dilenge and Ascherl [20] examined 3500 cerebral angiography images and Perrini et al. [14] dissected 14 adult cadaveric orbits and they showed that all recurrent meningeal branches originated from the common trunk of the OA. In our study, 40 of 47 recurrent meningeal branches $(85.1 \%)$ arose directly from the LA, 4 (8.5\%) from the anastomotic branch with MMA, and 3 (6.4\%) were directly derived from the OA. Erdoğmuş and Gövsa [24] measured the mean diameter of recurrent meningeal branch $0.85 \pm 0.25 \mathrm{~mm}$ on the right and $0.90 \pm 0.14 \mathrm{~mm}$ on the left. In our study, the mean diameter was found to be $0.21 \pm 0.06 \mathrm{~mm}$, and it was lower than that reported in the study of Erdoğmuş and Gövsa [24].

Diamond [2] performed a series of 13 orbital dissections, representing 10 individuals. He selected only orbits that indicated an artery passing through the Hyrtl canal. These specimens were more likely to demonstrate the double arterial route. He detected that the LA arose from the anastomotic branch with MMA in 7 of 13 orbits whereas we found this condition in 14 of 15 angiographic images. The rate of the LA originating from this anastomotic branch was found higher in our study than in Diamond's work. Dual persistence of the recurrent meningeal branch and the anastomotic branch with MMA was observed in 4 of 13 orbits by Diamond [2] and in 2 of 14 orbits by Perrini et al. [14]. Similar to the works of Diamond [2] and Perrini et al. [14], 
we detected the presence of these two branches in 4 angiographic images simultaneously.

\section{Anterior falx artery}

Anterior falx artery has been first described by Kuru [25] in 1967 using ICA angiographies. It enters between the two layers of the falx cerebri, usually over the cribriform plate $[10,25,26]$. It can be demonstrated on both sides, but one side may be dominant in the blood supply of the falx. It may be difficult to differentiate angiographically between the anterior meningeal artery and the anterior falx artery. Anterior falx artery lies close to the midline and courses straightly in antero-posterior view, whereas anterior meningeal artery courses a zig-zag and separates from the midline. Anterior meningeal artery sometimes follow a groove on inner surface of frontal bone. In many cases of ICA occlusion, the anterior meningeal branches and the anterior falx artery are filled through their anastomoses with the MMA [25]. In our study, meningeal branch of the anterior ethmoidal artery courses straightly and lies near to the midline. So, we accepted the meningeal branch of the anterior ethmoidal artery as an "anterior falx artery".

Pollock and Newton [27] visualized the anterior falx artery by injecting Micropaque into the ICA in cadavers. They showed that this vessel was a paired, slightly sinuous vessel within the falx cerebri, closely related to its attachment to the dura of the convexity. They analyzed 274 ICA angiograms of 160 patients (age range 3-78 years) to determine the frequency of visualization of this artery. They detected normal anterior falx artery in 14 patients $(8.7 \%)$. In 11 of these 14, this vessel was extremely small and could be observed only a few centimeters above the cribriform plate [27]. In our study, we detected anterior falx artery in 86 of 126 angiographic images (68.3\%), which was higher than the work of Pollock and Newton [27]. Our result may be due to the use of superselective angiographic images. The mean diameter of the anterior falx artery was found to be $0.22 \pm 0.06 \mathrm{~mm}$ in our study, but we could not compare because there was no data in the literature.

\section{Clinical importance}

Knowledge of these anastomotic routes between the OA and the MMA can be useful when neurointerventional radiologists try to find an alternative way to deliver drugs to the eye and the OA cannot be catheterized [28]. Anastomoses between the OA and the MMA present an exact advantage when the researchers try to establish collateral pathways after acute occlusion of the OA, they preserve the vision in almost $85 \%$ of patients [29]. On the other hand, the same pathways may cause undesirable passage of embolic material into the OA and ultimately results in blindness [6, 22].

\section{Conclusion}

The anastomoses between the OA and the MMA are quite important clinically. Knowledge of these anastomotic routes is important to help the neurointerventional radiologists to avoid embolic eye complications and to find an alternative way to deliver drugs to the eye. These pathways are all essential for neurosurgeons to perform safe surgical procedures involving orbit.

Acknowledgements A partial grant from the Turkish Academy of Sciences was used for the analysis software in this study. The authors would like to thank Ms. Hale Akdemir (DT) for illustration.

Authors contributions H Akdemir Aktaş: Project development, data collection, manuscript writing, KM Ergun: Project development, data collection, manuscript writing and editing, İ Tatar: Project development, data analysis, manuscript editing, A Arat: Data analysis, manuscript editing, KM Hayran: Data analysis, statistical evaluation, manuscript editing.

Funding No financial support.

\section{Compliance with ethical standards}

Conflict of interest The authors declare that they have no conflict of interest.

\section{References}

1. Standring S (2016) Gray's anatomy - the anatomical basis of clinical practice In. Forty-first edn. Elsevier, USA, pp 439-440

2. Diamond MK (1991) Homologies of the meningeal-orbital arteries: a reappraisal. J Anat 178:223-241

3. Snell RS (2010) Clinical neuroanatomy, 7th edn. Lippincott Williams and Wilkins, Philadelphia, pp 432-433

4. Moore KL, Dalley AF, Agur AMR (2014) Clinically oriented anatomy, 7th edn. Lippincott Williams and Wilkins, Philadelphia, pp 871-872

5. Bonasia S, Bojanowski M, Robert T (2019) Embryology and anatomical variations of the ophthalmic artery. Neuroradiology. https ://doi.org/10.1007/s00234-019-02336-4

6. Geibprasert S, Pongpech S, Armstrong D, Krings T (2009) Dangerous extracranial-intracranial anastomoses and supply to the cranial nerves: vessels the neurointerventionalist needs to know. Am J Neuroradiol 30:1459-1468. https://doi.org/10.3174/ajnr. A 1500

7. Lasjaunias P, Berenstein A, Brugge KG (2001) Surgical neuroangiography-clinical vascular anatomy and variations. vol 1 . Second edition edn. Springer, New York, pp 426-455

8. Lasjaunias P, Vignaud J, Hasso AN (1975) Maxillary artery blood supply to the orbit: normal and pathological aspects. Neuroradiology 9:87-97

9. Hayreh SS (2006) Orbital vascular anatomy. Eye (Lond) 20:1130 1144. https://doi.org/10.1038/sj.eye.6702377 
10. Rhoton AL Jr (2002) The orbit. Neurosurgery 51:303-334

11. Bertelli E, Regoli M, Bracco S (2017) An update on the variations of the orbital blood supply and hemodynamic. Surg Radiol Anat 39:485-496. https://doi.org/10.1007/s00276-016-1776-9

12. Mames RN, Snady-McCoy L, Guy J (1991) Central retinal and posterior ciliary artery occlusion after particle embolization of the external carotid artery system. Ophthalmology 98:527-531. https ://doi.org/10.1016/S0161-6420(91)32261-9

13. Marx F (1949) An arteriographic demonstration of collaterals between internal and external carotid arteries. Acta Radiol 31:155-160

14. Perrini P, Cardia A, Fraser K, Lanzio G (2007) A microsurgical study of the anatomy and course of ophthalmic artery and its possibly dangerous anastomoses. J Neurosurg 106:142-150

15. Padget DH (1948) The development of cranial arteries in the human embryo. Contrib Embryol Carneg Instn 212:205-262

16. Uchino A, Saito N, Takahashi M, Kozawa E, Mizukoshi W, Nakajima R, Okano N (2013) Persistent dorsal ophthalmic artery and ophthalmic artery arising from the middle meningeal artery diagnosed by MR angiography at 3 T. Surg Radiol Anat 35:775-782. https://doi.org/10.1007/s00276-013-1085-5

17. Georgiou C, Cassell MD (1992) The foramen meningo-orbitale and its relationship of the development of ophthalmic artery. $\mathrm{J}$ Anat 180:119-125

18. Govsa F, Erturk M, Kayalıoglu G, Pinar Y, Ozer M, Ozgur T (1999) Neuro-arterial relations in the region of the optic canal. Surg Radiol Anat 21:329-335

19. FCoA T (1998) Terminologia Anatomica International Anatomical Terminology. Thieme, Stuttgart, Germany

20. Dilenge D, Ascherl GF (1980) Variations of the ophthalmic and middle meningeal arteries: relation to the embryonic stapedial artery. Am J Neuroradiol 1:45-53

21. Hayreh SS, Dass R (1962) The ophthalmic artery I-Origin and intra-cranial and intra-canalicular course. Br J Ophthalmol 46:65-98
22. Bracco S, Venturi C, Leonini S, Romano DG, Cioni S, Vallone IM, Gennari P, Hadjistilianou T, De Francesco S, Bertelli E (2016) Transorbital anastomotic pathways between the external and internal carotid systems in children affected by intraocular retinoblastoma. Surg Radiol Anat 38:79-87. https://doi.org/10.1007/ s00276-015-1519-3

23. Liu Q, Rhoton AL Jr (2001) Middle meningeal origin of the ophthalmic artery. Neurosurgery 49:401-407

24. Erdogmus S, Govsa F (2005) Importance of the anatomic features of the lacrimal artery for orbital approaches. J Craniofac Surg 16:957-964

25. Kuru Y (1967) Meningeal branches of the ophthalmic artery. Acta Radiol Diagn (Stockh) 6:241-251

26. Hayreh SS (1962) The ophthalmic artery III-Branches. Br J Ophthalmol 46:212-247

27. Pollock JA, Newton TH (1968) The anterior falx artery: normal and pathologic anatomy. Radiology 91:1089-1095

28. Bertelli E, Leonini S, Galimberti D, Moretti S, Tinturini R, Hadjistilianou T, De Francesco S, Romano DG, Vallone IM, Cioni S, Gennari P, Galluzzi P, Grazzini I, Rossi S, Bracco S (2016) Hemodynamic and anatomic variations require an adaptable approach during intra-arterial chemotherapy for intraocular retinoblastoma: alternative routes, strategies, and follow-up. Am J Neuroradiol 37:1289-1295. https://doi.org/10.3174/ajnr.A4741

29. Ahn JH, Cho YD, Kang HS, Kim JE, Cho WS, Jung SC, Kim CH, Han MH (2014) Endovascular treatment of ophthalmic artery aneurysms: assessing balloon test occlusion and preservation of vision in coil embolization. Am J Neuroradiol 35:2146-2152. https://doi.org/10.3174/ajnr.A3999

Publisher's Note Springer Nature remains neutral with regard to jurisdictional claims in published maps and institutional affiliations. 